\title{
The usefulness of the Minnesota Multiphasic Personality Inventory (MMPI-I) in predicting dropout from Cognitive Behavioural
} Therapy (CBT)

\author{
Panagiota Goga*1, Lefteris Konstandinidis², Thomai Lioura ${ }^{1}$, Agis Gouzaris ${ }^{3}$, \\ Nikolas Nikolaidis ${ }^{4}$ and Gregoris Simos ${ }^{4}$
}

\begin{abstract}
Address: ${ }^{1}$ Association for Mental Health and Social Rehabilitation (EPSIKA), Thessaloniki, Greece, ${ }^{2}$ Private Practice, Thessaloniki, Greece, ${ }^{3} \mathrm{CMHC}$ of Vyronas-Kessariani, Athens, Greece and ${ }^{4} \mathrm{CMHC} /$ Central District of Thessaloniki

* Corresponding author
\end{abstract}

from International Society on Brain and Behaviour: 3rd International Congress on Brain and Behaviour

Thessaloniki, Greece. 28 November - 2 December 2007

Published: 17 April 2008

Annals of General Psychiatry 2008, 7(Suppl I):SI I5 doi:I0.II86/I744-859X-7-SI-SII5

This abstract is available from: http://www.annals-general-psychiatry.com/content/7/SI/SII5

(c) 2008 Goga et al.; licensee BioMed Central Ltd.

\section{Background}

Although treatment outcome relates directly to adherence to therapy, we do not know much on what kind of patients will remain in or dropout from treatment. Increased scores in the Paranoid "Pa" MMPI-I subscale seem to be a prognostic factor of dropout from therapy. Low scores in the " $\mathrm{K}$ " adjustment MMPI-I subscale also seem to be related to premature dropout from therapy. Also Patients with 1-3/3-1 and 2-7/7-2 combinations had significantly more dropout rates.

\section{Materials and methods}

Aim of the present study was to investigate personality factors that could predict dropout from Cognitive Behavioural Therapy using the three-point combinations of the ten clinical scales of Minnesota Multiphasic Personality Inventory (MMPI-I).

We studied 102 consecutive patients, with a variety of DSM-IV diagnoses, referred to a clinical psychologists' private practice and treated with CBT. Seventy-two (73.5\%) patients completed CBT while twenty-seven patients (26.5\%) dropped out of treatment early. All patients had completed MMPI-I just after their intake and evaluation interview. Treatment completers and treatment dropouts were compared using the 1-2-3, 1-3-4, 2-3-4, 2-4-7, 4-7-8 \& 6-7-8 combinations, which are the more frequent com- binations of the ten clinical scales of MMPI-I. Chi-square test (x2) was used.

\section{Results}

Not even one of the three-point combinations of the ten clinical scales of MMPI-I could predict treatment discontinuation.

\section{Conclusions}

Although MMPI-I is a well-known and widely used instrument in the assessment of personality; the three-point combinations of the ten clinical scales can't be used to predict patients in high risk of dropping out of therapy.

\section{References}

I. Konstandinidis L., Goga Y., Lioura T., Gouzaris A., Nikolaidis N., Simos G.: Predicting Drop-Out From CBT Using The TwoPoint Codes Of The Minnesota Multiphasic Personality Inventory (MMPI). Poster presentation in the 36th Annual Congress of European Association for Behavioural and Cognitive Therapies, Paris, France, September 20062006.

2. Goga Y., Lioura T., Gouzaris A., Konstandinidis L.: Personality Factors Associated With Dropping Out Of Cognitive Behavioural Treatment. Poster presentation in the 2nd International Congress on BRAIN AND BEHAVIOUR: International Society on Brain and Behaviour. Thessaloniki, Greece, November 20052005.

3. Duckworth J., Anderson W.: MMPI Interpretation Manual For Counselors And Clinicians. Third edition edition. Accelerated Development INC; 1986. 